DEPIGENETICS

\title{
Testing the generality of non-genetic inheritance
}

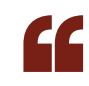

It will be interesting to test the pervasiveness of other proposed mechanisms of non-genetic inheritance

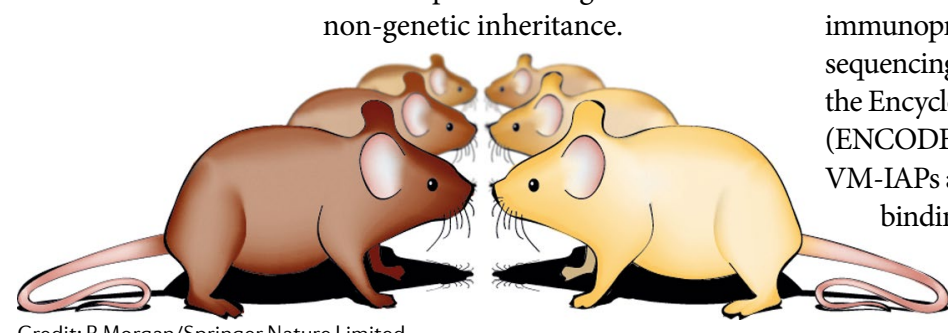

Credit: P. Morgan/Springer Nature Limited
Demonstrations of non-genetic inheritance attract considerable interest, but only a few robust, paradigmatic examples exist, which are often specific to particular species or genomic loci. Thus, a key issue is the extent to which these individual examples are applicable across additional genomic loci. A new study reports a mouse screen and concludes that non-genetic inheritance is likely to be the exception rather than the rule.

Kazachenka, Bertozzi et al. sought to understand whether two classic non-genetic inheritance paradigms in mice, at the Agouti viable yellow $\left(A^{\mathrm{vy}}\right)$ and Axin Fused $\left(A x \mathrm{xin}^{\mathrm{Fu}}\right)$ loci, are applicable to other genomic regions. These loci harbour an insertion of an intracisternal A particle (IAP) class of endogenous retroviral element (ERV). DNA methylation of the IAP is incomplete, leading to abnormal expression of the affected gene and a resulting phenotypic change in coat colour (for $A^{\mathrm{vy}}$ ) or tail shape (for Axin $^{\mathrm{Fu}}$ ). Crucially, the epigenetic state of these loci is metastable: sufficiently stable to be consistent between tissues of an individual, but unstable enough to be variable among individuals of an isogenic population. Despite the ability of the alleles to exhibit variable epigenetic states, the parental states and resultant phenotypes influence that of the offspring; hence, these are examples of transgenerational non-genetic inheritance.
As the C57BL/6J mouse strain studied by the authors has $\sim 12,000$ IAP-class ERVs in its genome, they screened for IAP insertions that exhibit equivalent properties to the IAPs at the $A^{\text {vy }}$ and $A x{ }^{\text {Fu }}{ }^{\text {loci. They }}$ devised a computational pipeline to analyse DNA methylation data (that they had generated as part of the BLUEPRINT reference epigenome project) for potential epigenetically metastable IAP alleles, whereby an IAP shows variability in DNA methylation signal between biological replicates of lymphocyte samples pooled across mice. Experimental validation showed that the identified IAPs had variable methylation levels across different mice, but consistent levels within the same mouse. Applying the analysis to IAPs genome-wide, the authors found that most IAPs were highly methylated across all data sets (consistent with the overall need to silence transposable elements to preserve genomic stability) but that $\sim 100$ were candidate variably methylated IAPs (VM-IAPs).

Among this set of candidate VM-IAPs, there were no strongly distinguishing sequence features that might be determinants of variable methylation, and the VM-IAPs represented the full range of IAP structures, from full-length IAPs to highly truncated forms. However, VM-IAPs were enriched for young (recently integrated) IAPs. Furthermore, analysis of chromatin immunoprecipitation followed by sequencing (ChIP-seq) data sets from the Encyclopedia of DNA Elements (ENCODE) project showed that most M-IAPs are bordered by CTCF binding peaks, with CTCF peaks closer to VM-IAPs than non-variable IAPs. As CTCF preferentially binds to unmethylated binding sites, the authors posit that antagonistic interplay between IAP methylation and CTCF binding site hypomethylation might be involved in the establishment or maintenance of VM-IAPs.

For these candidate VM-IAPs to exhibit analogous properties to the $A^{\text {vy }}$ and $A x i n^{\mathrm{Fu}}$ loci, their epigenetic state would need to alter the expression of nearby genes, and this state would be heritable. De novo transcriptome assemblies showed that only five VM-IAPs had evidence of initiating transcription into neighbouring genes (the mechanism by which the $A^{\text {vy }}$ and $A x i^{\mathrm{Fu}}$ IAPs alter local gene expression), with DNA methylation inversely correlated with expression levels. Additionally, one-third of the VM-IAPs overlapped endogenous transcripts, but the effect on host gene expression is currently unclear.

Testing of organismal inheritance is not easily amenable to highthroughput analysis, but the authors tested six novel VM-IAPs, with only one, VM-IAP ${ }_{\mathrm{Gm} 13849}$, showing evidence that a parental methylation state could influence the state in offspring, and even then the effect was weak. In general, the methylation state of VM-IAPs was reprogrammed between generations, with offspring displaying the full range of methylation variability, without any apparent parental influence.

This important study does not refute the legitimacy of classic paradigms of non-genetic inheritance, but at least for mechanisms relying on IAP methylation states in mice, it indicates that these paradigms are likely to remain restricted to few examples. It will be interesting to test the pervasiveness of other proposed mechanisms of non-genetic inheritance (such as those involving germline RNAs) across further species.

Darren J. Burgess

ORIGINAL ARTICLE Kazachenka, A., Bertozzi, T. M. et al. Identification, characterization, and heritability of murine metastable epialleles: implications for non-genetic inheritance. Cell https://doi.org/10.1016/j.cell.2018.09.043 (2018) 